\title{
Plasmon phenomena as origin of DC-current induced resistivity oscillations in two-dimensional electron systems
}

\author{
Jesús Iñarrea \\ Escuela Politécnica Superior, Universidad Carlos III,Leganes, Madrid,Spain and \\ Instituto de Ciencia de Materiales, CSIC, Cantoblanco,Madrid,28049,Spain.
}

(Dated: November 30, 2018)

We analyze theoretically the oscillations that the magnetoresistivity of twodimensional electron systems present when a high intensity direct current is applied. In the model presented here we suggest that a plasma wave is excited in the system producing an oscillating motion of the whole two-dimensional electron gas at the plasma frequency. This scenario affects dramatically the way that electrons interact with scatterers giving rise to oscillations in the longitudinal resistivity. With this theoretical model experimental results are well reproduced and explained.

PACS numbers: 
Magnetotransport properties of highly mobile two-dimensional electron systems (2DES) is a subject of increasing interest. The future nanoelectronics will integrate 2DES as key parts of different devices as transistors, diodes or capacitors. In particular the phenomena of microwave-induced resistivity oscillations (MIRO) and zero resistance states (ZRS) $1,2,3,4,5,6,7,8$ have attracted considerable attention both from theoretical and experimental sides. However the mechanism responsible is still under debate.

Another striking non-linear effect has been observed when a 2DES is subjected to a high intensity DC-current ${ }^{9}, 10,11$. These experiments report oscillations in the longitudinal magnetoresistivity $\left(\rho_{x x}\right)$ that are periodic in inverse magnetic field and tend to be qualitatively similar to the ones obtained in the experiments of microwave (MW) excited magnetoresitivity in Hall bars $1,2,3,4$. Another remarkable experimental outcome is that under DC-current excitation, the $\rho_{x x}$ response shifts towards higher magnetic fields $(B)$ with increasing DC-current intensity. Also the electron sheet density dependence shows that the maxima positions in the $\rho_{x x}$ oscillations are scaled as $1 / \sqrt{n_{e}}$ where $n_{e}$ is the electron sheet density. The first explanation to these effects $, 10,11$ considers that oscillations are related to elastic scattering mediated by a short range disorder between Hall fieldtilted Landau levels. Accordingly an electron may transfer momentum in the x-coordinate that is equivalent to a hopping in the y direction. $\rho_{x x}$ reflects this hopping through the conductivity $\sigma_{y y}$. Other theoretical proposals have been reported recently $\underline{12}$.

In this letter we present an alternative approach to explain these effects. In our model the DC-electric field $\left(E_{D C}\right)$, which produces the DC-current $\left(I_{D C}\right)$, displaces the orbit center of the harmonic quantum oscillators that are the electrons in the presence of a uniform and perpendicular $B$. This displacement with respect to the positive lattice ions disturbs the 2D electron gas leading to a collective excitation of the system. In other words, a plasma wave originates from a spatial shift of the 2DES as the charge seeks to move to restore equilibrium. The system responds with an oscillatory motion where the frequency is denoted as $w_{p}$ or plasma frequency. Evidently its effect is only measurable at sufficient high DC-current intensities. This scenario affects dramatically the way that electrons interact with scatterers and is reflected in the $\rho_{x x}$ response. We explain and reproduce the $\rho_{x x}$ oscillations periodicity in $B^{-1}$, the shift to larger $B$ with increasing 
current intensity and the dependence on $n_{e}$.

The electrons of a 2DES subjected to a perpendicular $B$, behave as harmonic quantum oscillators. Then if we apply a constant electric field $E_{D C}$ in the current direction (xdirection), we obtain a displacement $x$ of the center of all oscillators in the same direction: the entire $2 \mathrm{D}$ electron gas moves. The maximum value of $x$ is given by $\frac{13,14}{14} x_{\max }=\frac{e E_{D C}}{m^{*} w_{c}^{2}}$ where $e$ is the electron charge and $w_{c}$ the cyclotron frequency. The spatial shift of the electron gas as a whole with respect to the fixed positive background (lattice ions) give rise to two lines of opposite charge at either end of the sample creating an electric field $E_{p}$ (see Fig. 1). We obtain this field using basic Electrostatics $\frac{15}{\underline{E}}: \vec{E}(p)=\frac{n_{e} e x}{2 \pi \epsilon L_{e f}} \vec{i}$ being $L_{e f}$ the average effective distance between the two lines of opposite charge that we approximate by $L_{e f}=L-x_{\max }, \epsilon$ is the dielectric constant and $L$ the sample length. This field tends to restore the system to its equilibrium position producing in the system a collective excitation or plasma wave. Eventually the 2D electron gas obey the equation of motion of a harmonic oscillator ${ }^{16}$ that leads to oscillations at the plasma frequency $w_{p}$ :

$$
w_{p}=\sqrt{\frac{n_{e} e^{2}}{2 \pi m^{*} \epsilon L_{e f}}}
$$

On the other hand the plasma oscillation can be damped. Physically the coupling of the plasma to the lattice through phonon scattering results in a net decrease of the energy of the oscillating electron gas. The result is a progressive decay of the plasma oscillation being reflected in a continuous decrease of its amplitude. Eventually the plasma oscillations can be totally damped. However the external field $E_{D C}$ is still acting on the 2DES exciting the plasma wave and avoiding the oscillatory motion to stop. Thus $E_{D C}$ plays the role of and exciting force on the oscillating plasma. If we name this force as $F_{D C}$, the plasma equation of motion can be written as:

$$
N m^{*} \frac{d^{2} x}{d t}+N m^{*} \gamma \frac{d x}{d t}+N m^{*} w_{p}^{2} x=F_{D C}
$$

where $N$ is the number of electrons and $\gamma$ is a phenomenologically introduced damping factor which accounts for the electronic interactions with the lattice yielding acoustic phonons. To solve this differential equation we propose as a possible solution a harmonic function like $x_{p}=A_{D C} \cos w_{p} t$. The expression of $F_{D C}$ and the amplitude $A_{D C}$ are to be 
determined. Thus we obtain:

$$
x_{p}=\frac{e E_{D C}}{m^{*} \gamma w_{p}} \cos w_{p} t
$$

if $F_{D C}=N e E_{D C} \cos w t$ and $A_{D C}=\frac{e E_{D C}}{m^{*} \gamma w_{p}}$. Therefore we have a 2DES that oscillates as a whole with $w_{p}$, displacing the electrons orbit center with $x_{p}(t)$. The oscillations are excited by $F_{D C}$ and damped by coupling with the lattice.

Now we introduce the scattering suffered by electrons due to charged impurities randomly distributed in the sample. Without high intensity DC-current excitation, an electron in an initial state corresponding to an orbit center position $X_{n}^{0}$, scatters and jumps to a final state with orbit center $X_{m}^{0}$, changing its average coordinate in the static electric field direction in $\Delta X^{0}=X_{m}^{0}-X_{n}^{0}$. This is the relevant direction (x-direction) in this problem due to the presence of the DC electric field. It means that all the scattering jumps will be averaged to zero except the ones in the x-direction. Under a high intensity DC-current the plasma wave is excited and the electronic orbit center coordinates change being given, according to our model $\underline{13}, \underline{14}$, by:

$X^{p}=X^{0}+x_{p}=X^{0}+A_{D C} \cos w_{p} t$. Thus, due to the collective oscillation all the electronic orbit centers in the sample oscillate back and forth in the $\mathrm{x}$ direction through $x_{p}$. When an electron suffers a scattering process with a probability rate given by $W_{m, n}$, it takes a time $\tau=\frac{1}{W_{m, n}}$ for that electron to jump from an orbit to another. This probability rate is calculated according to the model described in ref ${ }^{13,14}$. If we consider that the electron jumps from the initial state oscillation middle position, and it takes a time $\tau$ to get to the final one, then we can write for the average coordinate change in the $\mathrm{x}$ direction: $\Delta X^{p}=\Delta X^{0}+A_{D C} \cos w_{p} \tau$ Finally the longitudinal conductivity $\sigma_{x x}$ can be calculated: $\sigma_{x x} \propto \int d E \frac{\Delta X^{p}}{\tau}\left(f_{i}-f_{f}\right)$, being $f_{i}$ and $f_{f}$ the corresponding electron distribution functions for the initial and final states respectively and $E$ energy. To obtain $\rho_{x x}$ we use the well-known tensor relation $\rho_{x x}=\frac{\sigma_{x x}}{\sigma_{x x}^{2}+\sigma_{x y}^{2}}$, where $\sigma_{x y} \simeq \frac{n_{e} e}{B}$.

In Fig. 2a, we present calculated $\rho_{x x}$ versus $B$ at a direct current $I_{D C}=300 \mu A$. We observe clear oscillations in $\rho_{x x}$ showing four peaks. The oscillations can be explained in similar terms as the ones obtained with MW excitation 13 (see Fig. 3). When no plasma wave is excited, electrons jump between fixed orbits and on average an electrons advances a distance $\Delta X^{0}$ (Fig. 3.a). When the DC-current excitation is on, orbits are not fixed 
and instead move back and forth through $x_{p}$ with the frequency $w_{p}$. When the orbits, due to plasma oscillation, are moving backwards during the scattering jump the electrons advance an average larger distance than the no plasma wave case: $\Delta X^{p}>\Delta X^{0}$. This corresponds to an increasing conductivity (Fig. 3b). When the entire electron gas is moving forward, during the jump the electron advances on average a shorter distance: $\Delta X^{p}<\Delta X^{0}$ (Fig. 3c). This corresponds to a decrease in the conductivity with respect to the case without DC-current excitation.

In Fig. $2 \mathrm{~b}$ we present calculated $\rho_{x x}$ versus $B^{-1}$. We observe that $\rho_{x x}$ is periodic in $B^{-1}$ in agreement with experiments $\frac{9}{10}, 11$ with an spatial period of $\delta$, (see figure). This is clearly shown in the inset where we present the inverse of peaks position, $B_{n}^{-1}$ versus the order of the peaks $n$. According to our model, $\rho_{x x} \propto \cos w_{p} \tau=\cos \frac{w_{p}}{K B} \frac{13}{\underline{B}}, K$ being a constant, i.e., $\rho_{x x}$ is periodic with $B^{-1}$. Thus, in the peaks the next condition is fulfilled: $B^{-1}=\frac{K}{w_{p}} 2 \pi n$. Therefore we obtain the equation of a straight line that crosses the origin as in experiments $\frac{9}{10}$.

In Fig. 4, we present calculated $\rho_{x x}$ versus $B$ for different values of $I_{D C}$. We observe that the peaks shift towards higher $B$ with increasing $I_{D C}$. An increasing $I_{D C}$ corresponds to a larger $E_{D C}$ and $x_{\max }$ affecting eventually $w_{p}$ (see eq. 2) that becomes larger too. Considering the functional dependence of $\rho_{x x}$ through $\cos w_{p} \tau$, a larger $w_{p}$ give rise to more peaks and a shift of $\rho_{x x}$ response to larger $B$. A similar behavior is obtained in MW-excited $\rho_{x x}$ response in Hall bars 1 . In Fig. 5, we present the calculated peaks position $B_{n}$ versus $n_{e}^{1 / 2}$. Two straight lines crossing the origin are obtained for peak order 1 and 2. The inset shows $\rho_{x x}$ versus $B$ for different $n_{e}$. Again following our model, $\rho_{x x} \propto \cos \frac{w_{p}}{W_{m, n}}=\cos C \frac{n_{e}^{1 / 2}}{n_{e} B}=\cos \frac{C}{n_{e}^{1 / 2} B} \frac{13}{13}, C$ being a constant. Peaks maxima fulfill $2 \pi n=\frac{C}{n_{e}^{1 / 2} B} \Rightarrow B=\frac{C}{2 \pi n} \frac{1}{n_{e}^{1 / 2}}$. As in the experimental outcome $\mathrm{e}^{9}$, we obtain the equation of a straight line crossing the origin.

This work has been supported by the MCYT (Spain) under grant MAT2005-06444, by the Ramón y Cajal program and by the EU Human Potential Programme: HPRN-CT2000-00144. 
1 R.G. Mani, J.H. Smet, K. von Klitzing, V. Narayanamurti, W.B. Johnson, V. Umansky, Nature 420646 (2002).

2 M.A. Zudov, R.R. Du, N. Pfeiffer, K.W. West, Phys. Rev. Lett. 90046807 (2003).

3 S.A. Studenikin, M. Potemski, A. Sachrajda, M. Hilke, L.N. Pfeiffer, K.W. West, Phys. Rev. B, 71, 245313, (2005).

4 A. A. Bykov, A. K. Bakarov, D. R. Islamov, A. I. Toropov, JETP Letters, 84 391, (2006).

5 C.Joas, J.Dietel and F. von Oppen, Phys. Rev. B 72, 165323,

6 M. Torres and A. Kunold, Phys. Rew. B. 71, 115313, (2005)

7 Assa Auerbach and G. Venketeswara, cond-mat/0612469.

8 RG. Mani, JH. Smet, K. von Klitzing, V. Narayanamurti, WB. Johnson and V. Umanski, Phys. Rev. Lett., 92, 146801, (2004).

9 C.L. Yang, R.R. Du, J.A. Simmons and J.L. Reno, Phys. Rev. Lett. 89, 076801 (2002).

10 A.A. Bykov, J. Zhang, S. Vitkalov, A.K. Kalagin and A.K. Bakarov, Phys. Rev. B, 72, 245397 (2005).

11 W. Zhang, H.S. Chiang, M.A. Zudov, L.N. Pfeiffer and K.W. West, Phys. Rev. B, 75, 041304(R) (2007)

12 X.L. Lei, Appl. Phys. Lett. 90, 132119 (2007)

13 J. Iñarrea and G. Platero, Phys. Rev. Lett. 94 016806, (2005); J. Iñarrea and G. Platero, Phys. Rev. B 72193414 (2005); J. Iñarrea and G. Platero, Appl. Phys. Lett. 89, 052109, (2006).

14 J. Iñarrea and G. Platero, Appl. Phys. Lett. 89, 172114, (2006); J. Iñarrea, Appl. Phys. Lett. 90, 172118, (2007); J. Iñarrea, Appl. Phys. Lett. 90, 262101, (2007); J. Iñarrea, G. Platero and C. Tejedor, Semicond. Sci. Tech. 9, 515, (1994); J. Iñarrea and G. Platero, Phys. Rew. B, 51, 5244, (1995).

15 R.K. Wangsness, Electromagnetics fields, 1st edition John Wiley ans Sons, New York. 1979.

16 N.W. Ashcroft and N.D. Mermin, Solid State Physics, 1 st edition, Saunders College, Philadelphia 1976. 
17 W. Zhang, M.A. Zudov, L.N. Pfeiffer and K.W. West, Phys. Rev. Lett. 98, 106804 (2007) 
Fig.1 caption: Schematic diagram showing electronic transport through a Hall Bar. A high intensity DC-current produces a displacement $x_{\max }$ of electrons orbit center with respect to the fixed positive background of lattice ions. The resulting linear distributions of opposite charge in opposite sides of the $2 \mathrm{D}$ sample give rise to an electric field $E_{P}$.

Fig.2 caption: (a) Calculated $\rho_{x x}$ versus $B$ at a direct current $I_{D C}=300 \mu A$. We observe clear oscillations in $\rho_{x x}$ response. Peaks order from 1 to 4 is shown. (b) Calculated $\rho_{x x}$ versus inverse magnetic field $\left(B^{-1}\right)$. The inset shows that $\rho_{x x}$ is periodic in $B^{-1}$, being $\delta$ the spatial period. $\mathrm{T}=1 \mathrm{~K}$.

Fig.3 caption: Schematic diagrams of electronic transport through the 2D sample without (a), (fixed orbits) and with plasmonic excitation (b) and (c), (oscillating orbits).

Fig. 4 caption: Calculated $\rho_{x x}$ versus $B$ for different values of $I_{D C}(50 \mu A \rightarrow 400 \mu A)$. We observe, as in experiments, that the peaks shift towards higher $B$ with increasing $I_{D C}$. The $\rho_{x x}$ shift and the increasing number of peaks with $I_{D C}$ suggest and increasing $w_{p} . \mathrm{T}=1 \mathrm{~K}$.

Fig. 5 caption: Calculated peak position $B_{n}$ versus $n_{e}^{1 / 2} . n_{e}$ is the electron sheet density. Two straight lines crossing the origin are obtained for peak order 1 and 2. The inset shows $\rho_{x x}$ versus $B$ for different $n_{e} . \mathrm{T}=1 \mathrm{~K}$. 

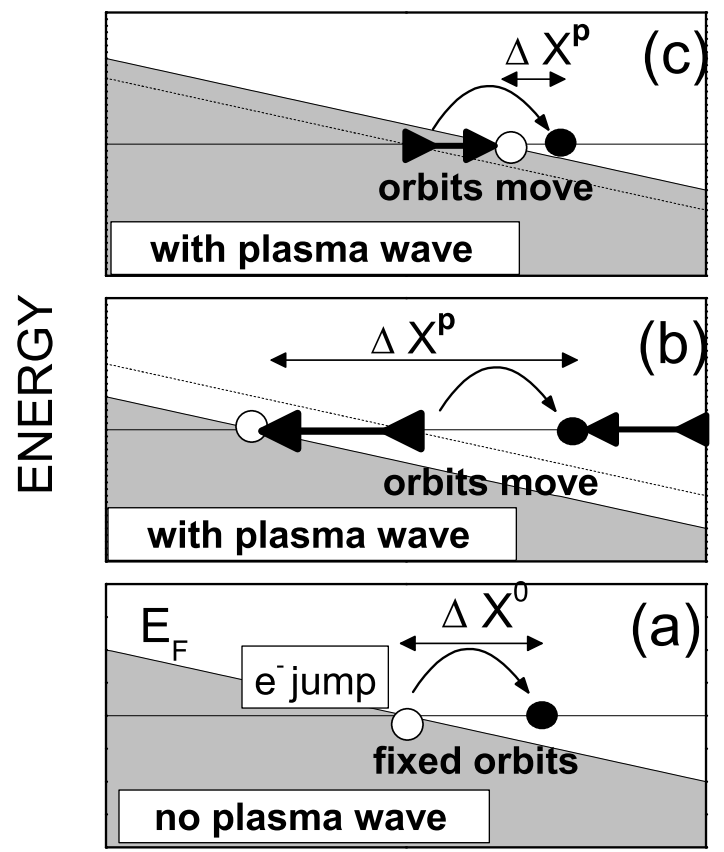

DISTANCE 

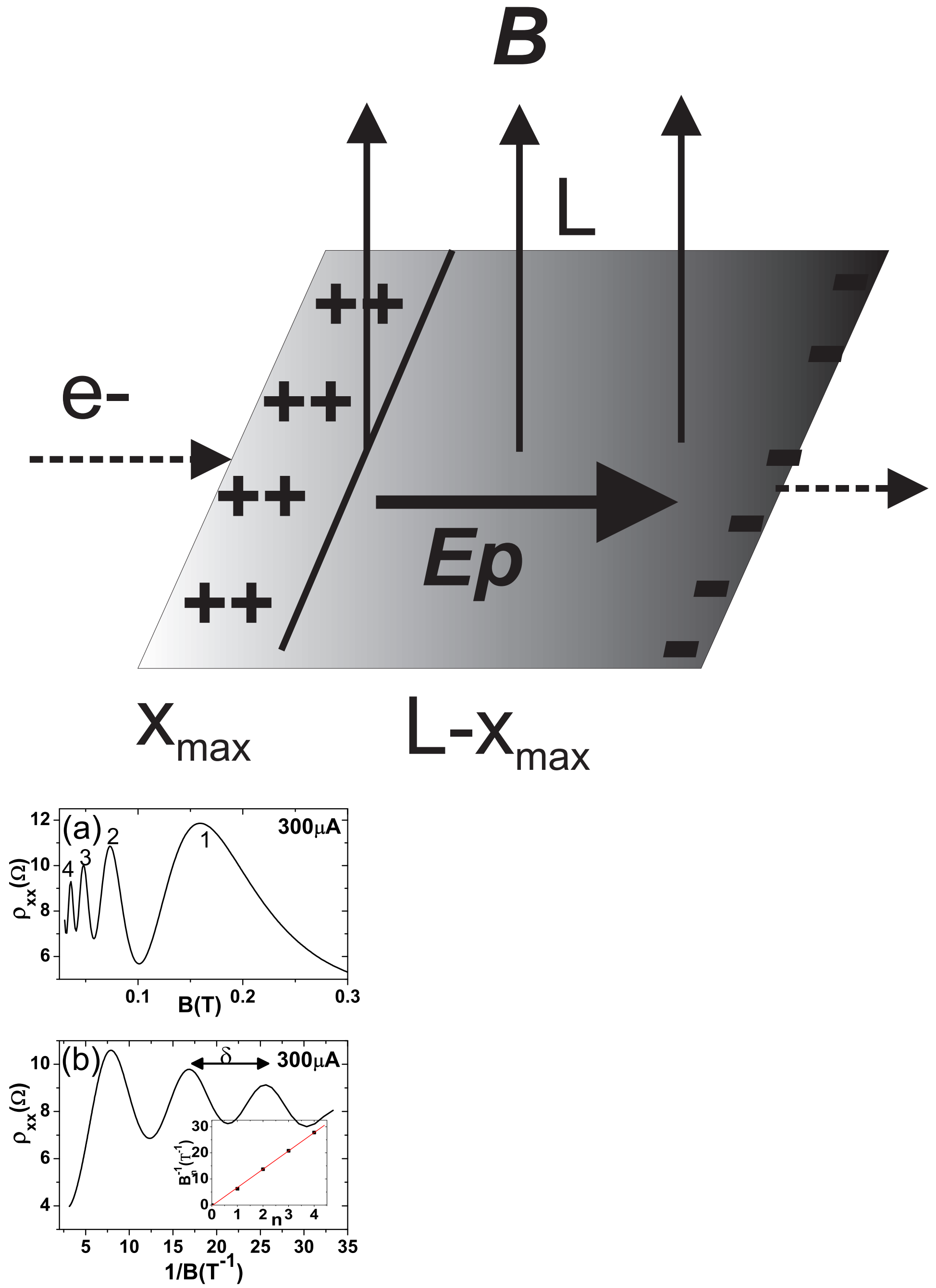


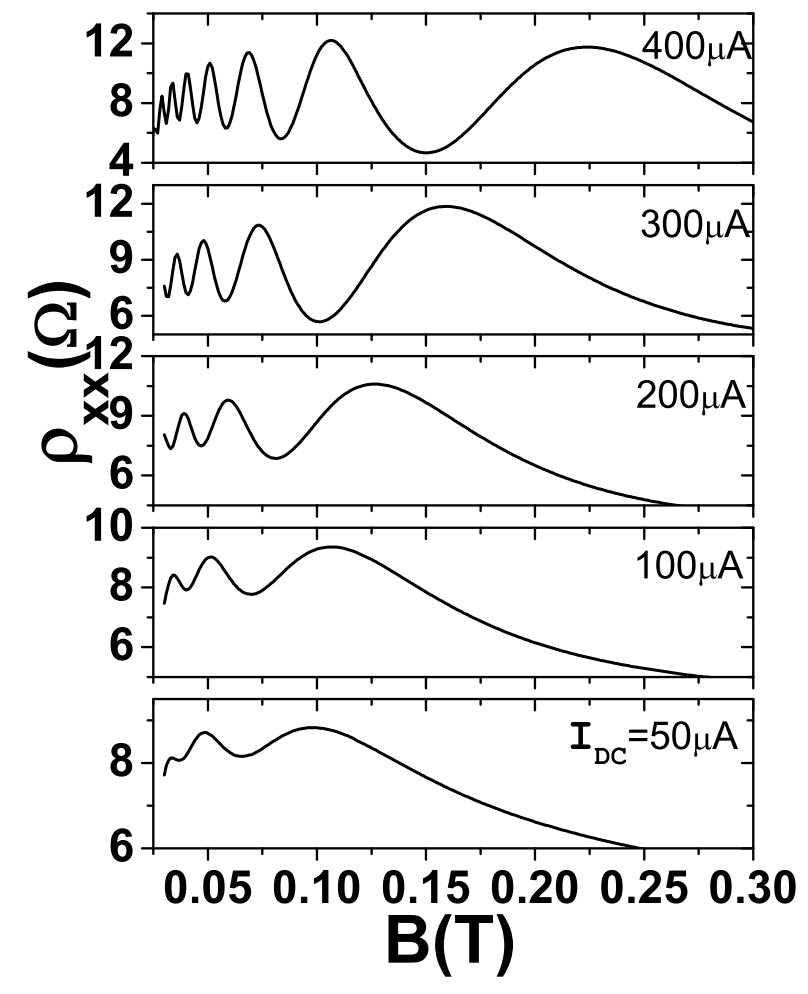




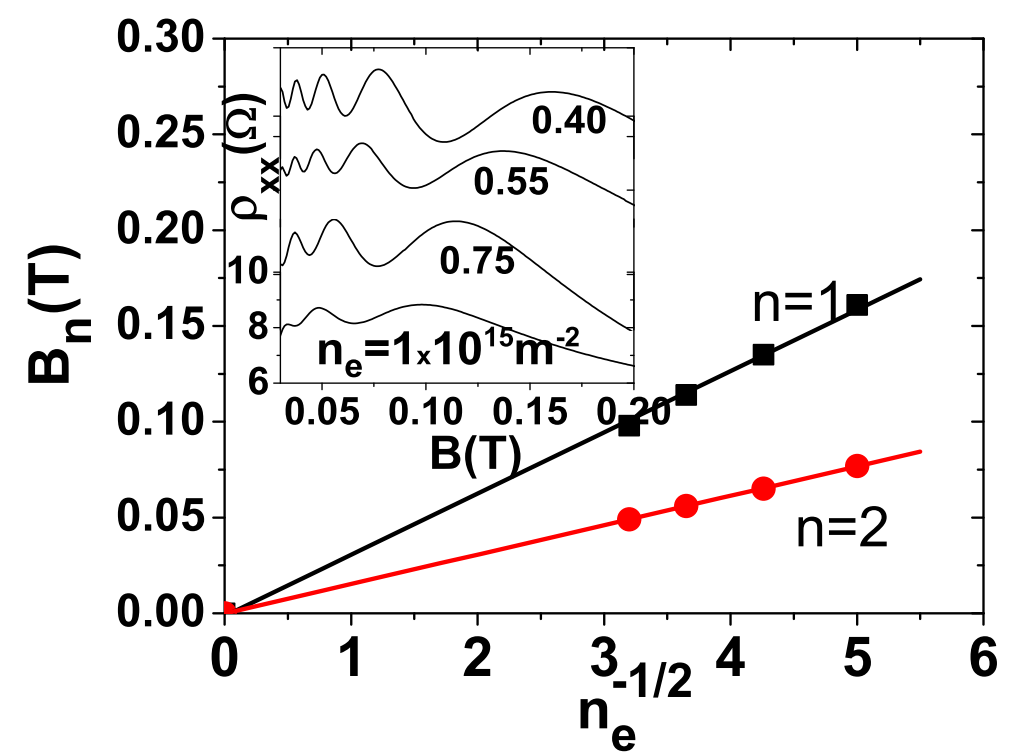

